\title{
XLVIII. An attempt to give a simple theoretical explanation of Raoult's law of the lowering of vapour-pressure
}

\section{F. G. Donnan}

To cite this article: F. G. Donnan (1892) XLVIII. An attempt to give a simple theoretical explanation of Raoult's law of the lowering of vapour-pressure, Philosophical Magazine Series 5, 34:210, 411-414, DOI: 10.1080/14786449208620353

To link to this article: http://dx.doi.org/10.1080/14786449208620353

曲 Published online: 08 May 2009.

Submit your article to this journal $\pi$

ЏII Article views: 3

Q View related articles $\longleftarrow$ 
Raouit's Law of the Lovering of Vapour-Pressure. 411 rotates. The plane-polarized rays into which the vibration may be resolved are represented as before by $\cos \omega t . \cos n t$. There is of course one case in which these complications fail to occur, i.e. when the axis of rotation coincides with the axis of vibration; but with axes distributed at random we must expect vibrations $(n \pm \omega)$ to be almost as important as the vibration $n$. The law of distribution of brightness in the spectral line would probably be exponential, as when the widening is due to motion of molecules as wholes in the line of sight.

It will be of interest to compare the magnitudes of the two effects. If $v$ be the linear velocity of a molecule and $V$ that of light, the comparison is between $\omega$ and $n v / V$, or between $\omega$ and $v / \lambda$. If $r$ be the radius of a molecule, the circumferential velocity of rotation is $\omega r$, and we may compare $\omega r$ with $v r / \lambda$. Now, according to Boltzmann's theorem, $r \omega$ would be of the same order of magnitude as $v$, so that the importance of the rotatory and linear effects would be somewhat as $\lambda: r$. There is every reason to suppose that $\lambda$ is much greater than $r$, and thus (if Boltzmann's relation held good) to expect that the disturbance of homogeneity due to rotation would largely outweigh that due to translation.

Your results seem already to interpose serious obstacles in the way of accepting such a conclusion; and the fact that light may thus be thrown upon a much controverted question in molecular physics is only another proof of the importance of the research upon which you are engaged.

I am,

Yours very truly,

Rayleigh.

September 23, 1892.

XLVIII. An Attempt to give a Simple Theoretical Explanation of Raoult's Law of the Lowering of Vapour-Pressure. By F. G. Donnan *.

RAOULT'S Law, as is well known, applies to the lowering 1 of the vapour-pressure of a liquid, due to the solution in it of a non-volatile substance. It asserts that the relative lowering of vapour-pressure is proportional to the ratio of the number of molecules of the dissolved substance to the total number of molecules in the liquid. Expressed in symbolical language this is

$$
\frac{f-f^{\prime}}{f^{\prime}}=k \frac{n}{\mathrm{~N}+n^{\prime}}
$$

* Communicated by the Author. 
where $f=$ original vapour-pressure, $f^{\prime}=$ reduced pressure, $n=$ number of molecules of dissolved substance, and $\mathrm{N}=$ number of molecules of solvent. The factor $k$ never deviates far from unity, and for solutions of medium concentration it is sensibly equal to unity, so that for such solutions we have

$$
\frac{f-f}{f}=\frac{n}{\mathrm{~N}+n} \text {. }
$$

This is the usual form in which Raoult's Law is applied. This equation has been theoretically deduced from the known laws of osmotic pressure by Van't Hoff and by Arrhenius. The following is an attempt to deduce it from the kinetic molecular theory.

Suppose we have a liquid in equilibrium with its vapour at a certain temperature $t$. Let the corresponding vapourpressure be $f$. We shall suppose the liquid and its vapour to be contained in a closed vessel, and we shall also suppose the temperature to remain constant, so that no permanent disturbance of equilibrium due to a permanent change of temperature need be considered. For this purpose we may imagine the walls of the containing vessel to be perfect conductors of heat, and suppose the vessel surrounded by a very large reservoir at constant temperature $t$.

This equilibrium of the liquid with its vapour is regarded as conditioned by a mutual and equal exchange of molecules. A certain definite number, $x$, of molecules escape per second from the superficial film of the liquid and pass into the vapour, while the same number, $x$, pass per second from the vapour into the liquid. Although the escape of any one particular molecule from the liquid into the vapour, and vice versa $\hat{a}$, is in itself a fortuitous occurrence, yet, owing to the very great number of molecules, a constant average is maintained and equilibrium preserved.

This is the usual view of liquid-vapour equilibrium, on the kinetic hypothesis.

Considering the vapour, $x$ will, cceteris paribus, be proportional to the number of molecules per unit volume-that is, proportional to the density, and therefore to the pressure. Thus we may write $x=c f, t$ being constant. The number of molecules in the liquid remains constant. Call this nnmber N. Now suppose we dissolve in the liquid $n$ molecules of a substance which exerts no appreciable vapour-pressure at the temperature $t, i$. $e$. whose molecules, for some reason or other, cannot escape at this temperature from the superficial film into the outer space. We have now a modified state of affairs within the liquid, It is allowable to suppose, as 
before, that $x$ molecules per second get an opportunity of escaping from the liquid, since there is nothing to alter this condition of things, as the molecules of the dissolved substance move freely about like those of the solvent. But, assuming a homogeneous composition of the solution, the theory of chances shows that only the fraction $\frac{\mathrm{N}}{\mathrm{N}+n}$ of the $x$ molecules which get suitable opportunities will be molecules of the solvent, and therefore able to escape. Thus we have now only $\frac{\mathrm{N}}{\mathrm{N}+n} x$ molecules escaping every second from the liquid.

Accordingly the previous mobile equilibrium is disturbed, for the liquid will now be gaining molecules, and the vapour losing them.

This will continue until the rates at which the liquid and vapour gain molecules again become equal. The value of the fraction $\frac{\mathrm{N}}{\mathrm{N}+n}$, however, changes during this process, inasmuch as $\mathrm{N}$ becomes $\mathrm{N}+v$, where $v$ is increasing and represents at any moment the number of molecules gained by the liquid (we must suppose that the film of pure solvent thus formed is dispersed and homogeneity secured by agitation of the liquid). Thus when equilibrium is again attained we shall have $\frac{\mathrm{N}+v}{\mathrm{~N}+n+v} x$ molecules escaping from the liquid into the vapour per second, and vice versâ. If $f^{\prime}$ is the new vapourpressure, we have now

$$
\frac{\mathrm{N}+v}{\mathrm{~N}+n+v} x=k f^{\prime}
$$

where $v$ has now its final value, $i$. $e$. the total number of molecules gained by the liquid. If we divide the members of this equation by the members of the previous equation, we obtain :-

$$
\frac{\mathrm{N}+v}{\mathrm{~N}+n+v}=\frac{f^{\prime}}{f}
$$

whence

$$
\frac{n}{\mathrm{~N}+n+v}=\frac{f-f^{\prime}}{f} \text {. }
$$

Now if the solution is dilute, $\frac{n}{\mathrm{~N}+n}$ will be a small fraction and 


\section{Raoult's Law of the Lowering of Vapour-Pressure.}

$v$ will be very small in comparison with $\mathrm{N}+n$. So that we may write, in accordance with Raoult's Law :- .

$$
\frac{n}{\mathrm{~N}+n}=\frac{f-f^{\prime}}{f^{\prime}} \text {. }
$$

For concentrated solutions, $v$ will be greater and $\frac{n}{\mathrm{~N}+n}$ will not be a small fraction, so that in this case it is just possible $v$ will have an appreciable effect, in which case we should have

$$
\frac{n}{\mathrm{~N}+n}=k \frac{f-f^{\prime}}{f^{\prime}},
$$

where $k$ is less than unity. But as $v$ will evidently never become of much importance in comparison with $\mathrm{N}+n, k$ will never deviate far from unity, as Raoult observed.

In the above investigation, $v$ stands for the number of molecules gained by the liquid. But it is evident $v$ is connected with the change of vapour-pressure. At first, number of molecules per unit volume of vapour $=\lambda f$. Denoting time by $t$, the rate at which the liquid gains molecules $=-\lambda \frac{d f}{d t}$. Hence the number of molecules gained by the liquid from volume $\mathrm{V}$ of vapour in time $t$ is expressed by

$$
-\nabla \lambda \int_{t=0}^{t=t} \frac{d f}{d t} d t=\mathrm{V} \lambda\left(f-f^{\prime}\right)=v .
$$

Thus we have :-

$$
\frac{n}{\mathrm{~N}+n+\mathrm{V} \lambda\left(f-f^{\prime \prime}\right)}=\frac{f-f^{\prime}}{f} .
$$

This expression assumes that the whole volume $V$ remains saturated.

Assuming that the fundamental equation of the kinetic theory of gases is applicable to the space occupied by the vapour (and the liquid surface acts towards the vaporous space just as if it were an immovable wall), it is easily seen that

$$
\lambda=\frac{3 v^{2}}{m},
$$

where $m=$ mass of a molecule, and $v=$ velocity of imean square of the molecules (the pressures $f$ and $f^{\prime}$ being measured in absolute units). 